Project Report

\title{
ERA-PLANET, a European Network for Observing Our Changing Planet
}

\author{
Kanaris Tsinganos 1,2,* Evangelos Gerasopoulos ${ }^{2}$, Iphigenia Keramitsoglou ${ }^{2}$, Nicola Pirrone ${ }^{3}$ \\ and the ERA-PLANET Team ${ }^{3}$ \\ 1 Department of Physics, National and Kapodistrian University of Athens, Athens 15784, Greece \\ 2 National Observatory of Athens, Athens 11810, Greece; egera@noa.gr (E.G.); ik@noa.gr (I.K.) \\ 3 Institute of Atmospheric Pollution Research, 7-00185 Rome, Italy; nicola.pirrone@iia.cnr.it \\ * Correspondence: tsingan@phys.uoa.gr; Tel.: +30-210-727-6915
}

Received: 29 April 2017; Accepted: 6 June 2017; Published: 16 June 2017

\begin{abstract}
ERA-PLANET is a wide European network comprised of 118 researchers from 35 partner institutions located in 18 countries, aiming to strengthen the European Research Area in the domain of Earth Observation (EO) in coherence with the European participation to the Group on Earth Observation (GEO) and the program for the establishment of a European capacity for Earth Observation, COPERNICUS. It will provide more accurate, comprehensive, and authoritative information to policy and decision-makers in key societal benefit areas (SBAs), under the umbrellas of dedicated projects in the topics of: smart cities and resilient societies; resource efficiency and environmental management; global changes and environmental treaties; polar areas and natural resources. ERA-PLANET will provide advanced decision support tools and technologies aimed to better monitor our global environment and share the information and knowledge in different domains of EO by launching joint transnational calls along the above four strands. The concept of the project that tackles with strand 1, as well as an example of a specific application fitting in, are described, aspiring to promote and coordinate the "smart-city" approach into a European network of cities and non-European follower cities, serving the need for a common approach to enhance environmental and societal resilience to air pollution, urban growth, and urban heat islands, as well as other natural/manmade stresses and relevant impacts. This is achieved through the synergy among technology, government, and society, while at the same time creating bridges between local/national initiatives with GEO/GEOSS, COPERNICUS, and other smart cities and GEO relevant projects. The project addresses initiatives in European cities but also specific issues dealing with air quality management in other parts of the world. Finally, it places major emphasis on fully exploiting key-enabling technologies and firmly addressing interoperability issues, in the context of big "smart city" data, and open science.
\end{abstract}

Keywords: earth observation; smart cities; GEOSS; air pollution; natural disasters; urban heat islands

\section{Introduction}

Sound and adaptive management of the Earth's natural resources and environmental quality requires reliable, relevant, and readily accessible scientific data. Several ground and space based instruments and facilities are increasingly used today to measure and monitor several specific aspects of Earth's physical, chemical, and biological systems. They include meteorological stations and balloons, computerized forecasting models, ocean buoys, seismic, and global positioning system (GPS) stations, remote-sensing satellites, early warning systems, etc. The raw data from those instruments must be processed, archived, interpreted, and finally made available in the form of useful information comprehensible not only by related experts and policymakers but also by society. During the last decade, the development of more powerful tools for data gathering and data sharing has increased 
the potential to foster the implementation of interoperable cyber infrastructures in environmental and EO sciences. This considerable amount of data has been rapidly made available to the scientific community, which has developed more analysis, methodologies, scientific assessments and has promoted technological progress. Despite these developments, however, this information is not always available in a suitable form for decision/policy makers. The vast amount of collected data have been archived in various systems, often not catalogued or indexed and not available in an integrated way to the general public or to policy makers and stakeholders. More efficient synergies between datasets would enhance new and innovative scientific and technical studies as well as fostering the use of these datasets by the general public and decision makers for technology and policy development. Recently, online data discovery and access (i.e., open data, data sharing) have become essential to enable multiand cross-disciplinary research for both the public and for decision makers. Making the best use of existing data and ensuring information system interoperability is a new important challenge.

Two aspiring projects in this direction of monitoring the Earth's environment are the international effort of the Group on Earth Observations (GEO) and the European Community's project COPERNICUS.

GEO, is a voluntary partnership of governments and international organizations established in 2005 and focusing on providing access to Earth observation data in several selected priority areas: climate change and its impacts, natural and human-induced disasters, environmental sources of health hazards, energy management, freshwater resources, weather forecasting, ecosystem management, sustainable agriculture, and biodiversity conservation. Furthermore, GEO coordinates international efforts to build a Global Earth Observation System of Systems (GEOSS) which links existing and planned Earth observation systems and supports the development of new ones in cases of gaps in the supply of environment-related information. GEOSS aims to construct a global public infrastructure for Earth observations consisting of a network of systems.

COPERNICUS is the world's largest single Earth Observation program which is directed by the European Commission in partnership with the European Space Agency (ESA) and aims at achieving a global, continuous, autonomous, high quality, wide ranging Earth Observation capacity. COPERNICUS provides an accurate, timely, and easily accessible information to improve the management of the environment, understand and mitigate the effects of climate change, and ensure civil security. It continues the work of the previous European Envisat program which operated from 2002 to 2012.

ERA-PLANET aims to bring together and strengthen the European, national, and regional R\&I programs in the domain of EO. The overarching goal of ERA-PLANET is the development of a Transnational Environmental Observation System in Support of European \& International Policies through the integration of real-time monitoring data from various platforms, modelling tools, and advanced global cyber-infrastructure for data sharing and interoperability. Specifically, ERA-PLANET intends to enhance its role in GEOSS by fostering a better cross- and interconnection of individual EO systems, ensuring a more efficient access and exploitation of datasets. In Europe, there is a clear need to establish a more effective interoperability between National and European research infrastructures in order to play a more strategic role in the Group on Earth Observations (GEO) and its Global Earth Observation System of Systems (GEOSS) initiative. The European Union (EU) through pursuing interoperability in the exchange of data, metadata, products and services in a full, open, and unrestricted manner would generate business opportunities for the private sector within the EU (SMEs, industries) leading to significant benefits to European society as a whole.

\section{Objectives}

The first major objective of the ERA-PLANET project is to prepare and launch a two-stage joint transnational call structured along the following four strands:

- Strand 1-Smart cities and resilient societies, addressing issues such as urban growth, air quality, disasters, health, contaminated sites. 
- Strand 2-Resource efficiency and environmental management, including water, energy, food security, biodiversity.

- Strand 3-Global change and environmental treaties, addressing global observing systems for toxic and persistent pollutants, harmonization of monitoring and coupled atmosphere-ocean-terrestrial models, evaluation of ecosystem response to regional/global emission changes, support to policy implementation, and climate forcing in assessing global change patterns and analysis of policy scenarios.

- Strand 4-Polar areas and natural resources in highly climate-sensitive regions, including the evaluation of the impact of energy resource exploitation, the impact of long-range transport of air pollutants and their atmospheric deposition, air-surface exchange mechanisms, and environmental pressure from increasing anthropogenic activity in areas with sensitive ecosystems.

ERA-PLANET subsequently will fund these projects according to a priority list set by external experts, further monitoring them and reporting on their progress. Additionally, ERA-PLANET will develop a strategic research agenda to reinforce the European research area and the cross- and inter-cooperation of European and national programs in key and selected EO domains and coordinate initiatives with the aim to improve the interoperability among existing and future projects on EO and links to the Global Cyber Infrastructure (GCI).

The project objectives exhibit the capacity of ERA-PLANET to reply to the key challenge of the call and specifically aim to simplify access to the information required by decision makers and bring together and strengthen the European national and regional research and innovation programs in the EO domain. ERA-PLANET will attempt to go beyond the coordination of EO during its implementation and will establish initiatives to continue after the duration of the project.

Finally, the project will benefit from the outcome of ongoing activities being carried out by several partners in the framework of international, national, and regional programs, including:

- UNEP Global Partnership on Atmospheric Mercury Transport and Fate Research (UNEP F\&T) (www.chem.unep.ch);

- Global Monitoring Plan (GMP) of the Stockholm Convention on Persistent Organic Pollutants (POPs) of UNEP (www.pops-gmp.org/);

- Task Force on Hemispheric Transport of Air Pollutants (TF HTAP) of the UNECE-LRTAP convention (www.htap.org);

- Several GEO flagships, initiatives, and tasks;

- ESFRI/I3 Research Infrastructures (e.g., ACTRIS-Aerosols, Clouds, and Trace gases Research InfraStructure Network, LTER, ANAEE, SIOS);

- National polar research programs involved in measurements devoted to climate forcing, biodiversity, ecosystem management, biogeochemical cycles of heavy metals in polar ecosystems, and evaluation of response times and mechanisms to changes in anthropogenic pressures and stressors.

Thus, the results of past and ongoing projects as well as other projects related to GEOSS and COPERNICUS developed within the framework of a number of GEO Tasks, will provide a solid basis of knowledge in terms of state-of-the-art atmospheric models, monitoring methodologies, QA/QC systems, interoperable management tools, and environmental policy analysis instruments and exploitation of COPERNICUS services. ERA-PLANET will also benefit from the outcomes of ongoing projects such as ENVRIPLUS because the overarching goal of these projects is to bring together networks on Environmental and Earth System Research Infrastructures and build common synergic solutions.

\section{Specific Targets}

Among the main ERA-PLANET targets is to address the need of policy makers and stakeholders for timely access to high quality data and tools on Earth Observation for developing innovative and 
competitive activities in the public and private sectors leading to an increase in competitiveness in the world market. The way that ERA-PLANET will answer the demand for interoperable, pervasive and prompt data availability is the brokering approach. All strands will be constructed in order to discover available datasets and services with this approach in mind. Each funded project will be required to develop a data infrastructure implementing a set of principles (e.g., GEOSS Data Sharing and Management principles, the DAB guidelines to contribute to GEOSS, the data policy guidelines for H2020). In the field of each ERA-PLANET strand the specific projects that will be funded through the ERA-PLANET initiative will be required to analyze current research and innovation programs on Earth Observation, define the fragmentation level and the possibility of integration in order to reach the critical mass necessary to reinforce the European contribution to key areas of EO. Thus, the specific targets of ERA-PLANET are to:

- Improve the alignment of national and European research programs dealing with EO and data infrastructures (take-on-board national agendas);

- Improve interoperability, via a coordination action, among the existing and emerging data infrastructures which contribute to GEOSS and the GCI;

- Ensure additional funding and integration of existing and future ground-truth facilities to validate EO data;

- Foster capacity building of EO initiatives within environment, health, and human activity domains;

- Improve societal participation in the EO initiatives;

- Integrate to the extent possible the overarching goals of GEOSS and COPERNICUS for key environmental priority issues aiming to support the implementation of the European Environmental Policy in different EO domains;

- Exploit COPERNICUS Core Services and EU capabilities in the EO domain;

- Improve monitoring and access to information through advanced technologies;

- Foster technological development to deliver more timely information in order to address the demand for high quality data and decision support tools;

- Lay the ground to incorporate data from national and EU funded 'Citizen Observatories' programs;

- Promote the use of open specifications (i.e., international standards and community specifications) for data sharing;

- Contribute to the definition, with potential end-users (i.e., stakeholders, policy-makers, companies, local authorities), of key and society-relevant indicators based on the essential variables and promote their generation for monitoring processes related to the Earth system monitoring (i.e., EOVs-Essential Ocean Variables; EBVs-Essential Biodiversity Variables);

- $\quad$ Links with other JPIs (i.e., FACCE, Cultural Heritage, Oceans) to foster interoperability and data sharing.

\section{Expected Impact of the Action}

The expected impact of the action can be summarized to be the following:

- Reinforcement of the Group on Earth Observations (GEO) post-2016;

- Coordination and integration of major research and innovation programs on EO as a contribution to Global Earth Observations System of Systems (GEOSS) and COPERNICUS;

- Maximization of value and benefits of EO investments;

- Development of sustainable and interoperable observational, modelling, data assimilation, and prediction systems;

- Fostering a wider exploitation and use of EO derived information for the benefit of citizens' daily life (e.g., extreme events forecast, coastal erosion, water, and air quality);

- Support an effective implementation of environmental policies; 
- Improve and select effective environmental indicators for different end-users.

The impact will be performed via a systematic dissemination and exploitation of the results, to maximize audience and societal benefits, as well as via communication activities.

\section{Smart Cities and Resilient Societies}

According to the UN's “World Urbanization Prospects: The 2014 Revision" in today's increasingly global and interconnected world, over half of the world's population lives in urban areas, while the coming decades will bring further profound changes to the size and spatial distribution of the global population. Continuing urbanization is projected to add 2.5 billion people to the urban population by 2050 , and the proportion of the world's population living in urban areas is expected to increase to $66 \%$. Under these circumstances, sustainable development challenges will be increasingly concentrated in cities (United Nations, Department of Economic and Social Affairs, Population Division (2014) (UN/WUP 2014) [1] To meet these challenges, cities and national governments around the world are investing in the potential of smart cities at an ever increasing rate: in China and India alone, almost 300 smart city pilots are currently planned, and the prediction for the global market for smart city technologies and services is $\$ 408$ billion by 2020 (BIS 2013) [2].

All aspects of future cities evolution demand for full exploitation of $\mathrm{EO}$ and new technologies towards building resilient societies and citizens and enabling informed decision making at the city level. Of course cities are quite complex environments in both economic, governance and societal terms and there might be numerous factors that may influence their ability to continue to function properly and coordinated, in the face of daunting and/or unexpected challenges, as a result of urbanization, climate change, or other potential stressors. Moreover, different cities may need to weigh vulnerabilities differently, based on the specific particularities they bear, and finally focus on different aspects that would help them bounce back rapidly from disruptions, exploiting advantages and assets already existing and/or applied. In this context, ERA-PLANET Strand 1 put emphasis on specific issues namely urban growth, air quality and urban microclimate, natural and manmade disasters, health, contaminated sites, and suggest specific case studies that present increasing interest in terms of physical processes (e.g., multiple and discrete pollution sources contributing to elevated pollution levels, complex terrains), urban morphologies and structural organization, high and uncontrolled urbanization growth rates, and vulnerability to climate change or the on-going economic crisis, the latter also resulting to behavioral changes.

The project to be launched under Strand 1, will bring together partners of a high degree of expertise in each of the fields addressed. The common, underlying thread that will effectively bind this vast array of disciplines and participants will be the "smart-city" concept, repurposed for addressing urban environmental pressures and building societal resilience. The consortium is gradually constructed after preparatory discussions in which the partners share the mutual belief that robust science, informed policy making, emergency planning, active and aware citizens is the optimal way forward for serving the strand's objectives and the particular objectives of the project.

The overall concept therefore is the refocusing of each partner's expertise and efforts under the "smart-city" concept. The refocusing is not arbitrary but will be based on current scientific knowledge, on current and future urban pressures and on stakeholders and citizens' needs that will be further designated within the project. The different methods and solutions that each partner will bring forward will constitute the backbone of the project and will not be disconnected. Inter- and trans-disciplinary interaction will be fostered throughout the duration of the project among the partners themselves and with the wider community of the Region of Interest (RoI) as well, so that a portfolio of integrated EO platforms/services/solutions is established, added value products are created, and key indicators are served. These different in situ, remote sensing, modelling, and innovative components will be the project's spearhead for fulfilling its objectives, while their integration will allow for cross-cutting along more entangled issues such as health and migration. The portfolio is of central importance and it is noted that the project aspires to be an initiative that will have a lasting and sustainable effect. To that 
end, extroversion is heavily built in the overall concept, with specific actions for interoperability (under the GEOSS and COPERNICUS framework), networking with relevant projects, replicability (pilot/test case and subsequent follower-cities) and transfer of knowledge in the RoI and other regions of the world facing similar pressures (Figure 1).

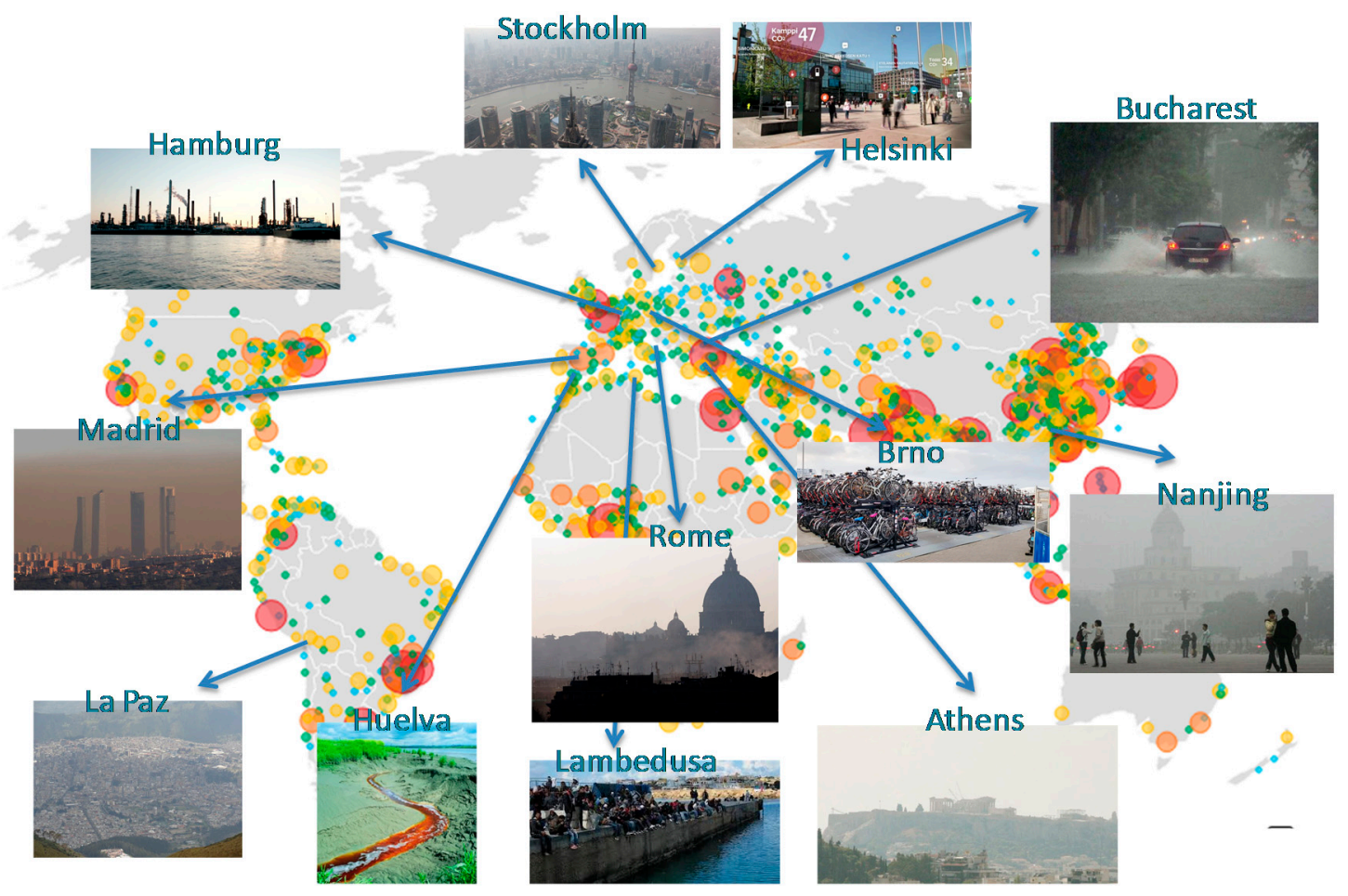

Figure 1. ERA-PLANET candidate pilot "smart cities".

The project will use the 'lighthouse city' approach (pilot cases), i.e., selected smart cities of the European network will set the stage to integrate and unify existing, increasing, but still fragmented EO resources (satellites, in situ networks/sensors, UAVs, models, citizen observatories), into information and decision making tools for individuals and local governments, tailored to the topics of the projects (air quality, disasters, urban growth). The partners will reach out to the different levels of the community and stakeholders to identify specific user needs. Either by existing projects' results or by new surveys, local authorities, urban planners, city level stakeholders, specific citizen communities, and humanitarian bodies will contribute to defining the urban pressures and the smart-city solutions that best fit their location and needs. Common patterns are expected to arise, later enabling clustering of cities based on actual needs.

The partners will bring mature methods and tools to the project-from the in situ, remote sensing, modeling, and other innovative observational platforms - that will be further refocused and fine-tuned to adjust to the city scale "terrain" and needs. Cities that meet specific criteria (e.g., in terms of urban pressure hierarchy, feasibility, capacity to integrate several solutions) will be designated as Lighthouse Pilot Cities, while Test-Bed Cities will complement this array of varying degree of implementation, focusing on a single issue for solution correspondence and its testing. Finally, there will be Follower Cities that observe and interact or even contribute to pilots' implementation, and during the project lifetime they pave the ground for future exploitation of its solutions. The design of the project will allow for needs and requirements to be clustered and classified allowing for optimum issue-to-solution correspondence and secondly, duplicate effort will be minimized. The project will also include case studies, during which EO will be exploited synergistically to tackle the more encompassing, 
complicated and sensitive, high priority EU agenda issues like indicatively health and the European migrant crisis.

\section{Urban Heat Islands}

Another challenge faced by urban areas is the Urban Heat Island (UHI) effect. This effect refers to the elevated temperatures of urban areas in respect to their surrounding rural areas and it is a result of changes in the local energy balance of urban areas caused by the conversion of pervious surface to impervious surfaces, the morphology of cities, and the increased anthropogenic heat fluxes, to name a few. The UHI effect is an important problem that can impact the health and comfort of the urban population; increase the energy demand of cities; and intensify and prolong heatwaves. The consequences of increased urban temperatures are most visible during heatwaves: a significant increase in heat-related deaths (e.g., the 2003 heatwave resulted to 25,000-70,000 excess deaths across Western Europe); the disruption of many socioeconomic activities due to thermal discomfort; and the possible occurrence of energy blackouts. These effects are not uniform across a city; but depend on many city specific characteristics such as land cover/land use, building conditions, and population characteristics (distribution, age, health conditions, mobility, poverty, and isolation).

Earth Observation can provide the tools to quantify most of these variables and thus facilitate the monitoring of urban areas (Sismanidis et al. [3]). Thermal Earth Observation data in particular can provide information about the urban thermal environment and the spatial distribution of temperatures (Sismanidis et al. [4,5]). At the moment, the collection of urban temperature data in most cities cannot meet the requirements in spatial and temporal resolution required for monitoring urban temperatures. This is because the installation and operation of dense weather station networks is very expensive; whilst the model-based solutions are less accurate and spatially coarser than required to capture the intra-city variabilities. To that end, Earth Observation can offer a simultaneous and continuous view of the urban thermal environment with minimum cost and form the basis for the estimation of several environmental parameters relevant to UHIs (Keramitsoglou et al. [6]) and also other higher level products as presented in Figure 2.

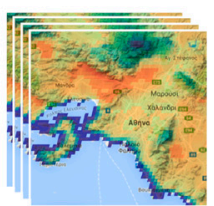

\section{$\mathbb{E}$ LST}

The skin temperature of the surface. Closely related to the surface energy balance.

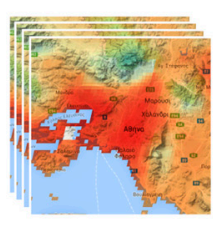

\section{ÉTA}

The air temperature

at $2 \mathrm{~m}$ above ground. Drives the heat transfer process of the human body.

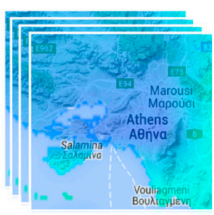

\section{go $\mathrm{RH}$}

Relative Humidity is the amount of water present in air expressed as a percentage.

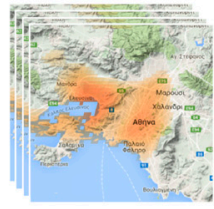

\section{$\Downarrow$ Couling Degrees}

The difference between TA and a threshold above which people use air-conditioning to sustain the indoor temperature to comfortable levels.
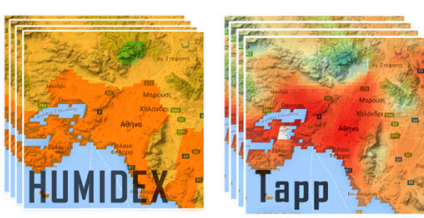

(6) HUMIDEX ¿ Tapparent

Peoples' discomfort due to temperature and humidity.

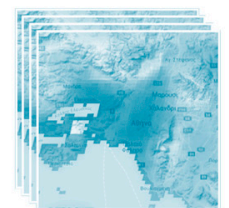

\section{(6) Heatwave Hazard}

TA discrepancy from a city specific threshold above which a day is considered Hot.

Figure 2. Several environmental parameters and higher level products that can be estimated from Earth Observation data and be used for the study of UHIs.

Hence, understanding, monitoring, and mitigating the UHI effect is a key so as to maintain an acceptable quality of life for the foreseeable future. To achieve this, urban areas have to be properly managed and Earth Observation is key for informing policy makers. In ERA-PLANET, 
we foresee the real time monitoring of the urban thermal environment of selected cities and support the development of innovative applications related to energy and health, by means of Application Program Interfaces (APIs).

Acknowledgments: The authors wish to acknowledge the contribution of the ERA-PLANET project (Grant agreement no. 689443) funded by the European Commission as part of the H2020-SC5.

Conflicts of Interest: The authors declare no conflict of interest.

\section{References}

1. United Nations, Department of Economic and Social Affairs, Population Division. World Urbanization Prospects: The 2014 Revision, Highlights (ST/ESA/SER.A/352). Available online: https:/ / esa.un.org/ unpd/wup/publications/files/wup2014-highlights.Pdf (accessed on 7 February 2017).

2. Department for Business, Innovation and Skills. The Smart City Market: Opportunities for the UK. Available online: https://www.gov.uk/government/uploads/system/uploads/attachment_data/file/249423/bis13-1217-smart-city-market-opportunties-uk.pdf (accessed on 12 May 2017).

3. Sismanidis, P.; Keramitsoglou, I.; Kiranoudis, C.T. A satellite-based system for continuous monitoring of surface urban heat islands. Urban Clim. 2015, 14, 141-153. [CrossRef]

4. Sismanidis, P.; Keramitsoglou, I.; Kiranoudis, C.T.; Bechtel, B. Assessing the capability of a downscaled urban land surface temperature time series to reproduce the spatiotemporal features of the original data. Remote Sens. 2016, 8, 274. [CrossRef]

5. Sismanidis, P.; Keramitsoglou, I.; Bechtel, B.; Kiranoudis, C.T. Improving the downscaling of diurnal land surface temperatures using the annual cycle parameters as disaggregation kernels. Remote Sens. 2017, 9, 23. [CrossRef]

6. Keramitsoglou, I.; Kiranoudis, C.; Sismanidis, P.; Zakšek, K. An Online System for Nowcasting Satellite Derived Temperatures for Urban Areas. Remote Sens. 2016, 8, 306. [CrossRef] 